\title{
The influences of anesthesia methods on some complications after orthopedic surgery: a Bayesian network meta-analysis
}

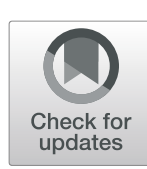

Yuqing Zeng ${ }^{1,2}$, Junming Wan $^{2}$, Haiyong Ren², Jianwei $\mathrm{Lu}^{2}$, Fuhua Zhong ${ }^{2}$ and Shu Deng ${ }^{3^{*}}$ (D)

\begin{abstract}
Background: Although several anesthesia procedures have been explored for orthopedic surgery, the complications of anesthesia remain not well resolved. This study aimed to explore the influence of different anesthesia methods on the complications after orthopedic surgery.

Methods: According to the searching strategy, anesthesia associated studies in orthopedic surgery were screened from Pubmed, Embase, and the Cochrane Library up to Mar. 10th, 2018. Then, complications and demographic data were extracted and quality of studies was assessed using Cochrane Collaboration recommendations. ADDIS software was used to perform the network meta-analysis. Pooled effect size was calculated using random effective model or consistency model, and presented with odds ratio (OR) and 95\% confidence interval (CI).

Results: According to the selective criteria, a total of 23 studies with 2393 patients were enrolled in this study. Quality assessment revealed all studies had an ordinary quality. Network meta-analyses revealed that nerve block analgesia (NBA) presented a lower effect on the occurrence of post-operative nausea or vomiting (PONV; $O R=0.17$, 95\% Cl: $0.06-0.39)$ and urine retention ( $\mathrm{OR}=0.07,95 \% \mathrm{Cl}$ : 0.01-0.37) compared with epidural anesthesia (EA). Interscalene block (ISB) and local infiltration analgesia (LIA) could significantly reduce the occurrence of back pain compared with $\mathrm{EA}(\mathrm{OR}=0.00,95 \% \mathrm{Cl}=0.00-0.30 ; \mathrm{OR}=0.00,95 \% \mathrm{Cl}=0.00-0.25)$.
\end{abstract}

Conclusion: NBA presented an effective role in reliving the occurrence of PONV and urine retention, and ISB and LIA relieved the back pain compared with EA after orthopedic surgery.

Keywords: Orthopedic surgery, Nerve block analgesia, Local infiltration analgesia, Interscalene block, Complication

\section{Background}

Since it emerged in the eighteenth century, the discipline of orthopedic surgery has been remarkably developed [1]. Till now, several orthopedic surgeries have been explored, including total knee replacement, hip fracture, and total hip replacement [2]. However, there are still some deficiencies to limit the application of orthopedic surgery in clinic, such as pain control, prevention of post-operative nausea or vomiting (PONV), rapid recovery, cognitive impairment, and surgical site infection [35]. Specifically, anesthesia is a common procedure during orthopedic surgery, which could affect the

\footnotetext{
* Correspondence: ShushuDeng55@outlook.com

${ }^{3}$ Department of Hematology, The First Affliated Hospital of Zhejiang Chinese Medical University, 54 Youdian Road, Hangzhou, Zhejiang Province, People's Republic of China

Full list of author information is available at the end of the article
}

temperature regulation, infection, bleeding, oxygen consumption, and other complications to influence the outcome of orthopedic surgery [6]. Therefore, it is important to innovate appropriate anesthesia manner to improve the outcomes and prognosis of orthopedic surgery.

With the development of few decades, although several anesthesia manners have been explored for orthopedic surgery, the complications of anesthesia are still not well resolved. A previous study has revealed that patients managed with general anesthesia perform a low risk of complications compared with patients undergoing spinal anesthesia during the total knee arthroplasty [7]. However, compared with the general anesthesia, regional anesthesia presents a better outcome than general anesthesia in total hip arthroplasty, including reductions 
of deep surgical site infection, length of hospital stay, and pulmonary complication [8]. Moreover, Stundner et al. have revealed that neuraxial anesthesia reduces the occurrence rates of blood transfusions and morbidity in the perioperative period of compared with general anesthesia for simultaneous bilateral total knee arthroplasty [9]. In addition, Ewan et al. have documented that general anesthesia increases the risk of post-operative cognitive dysfunction compared with other anesthesia methods [10]. Considering of these evidences, there is still no clear consensus in anesthesia during orthopedic surgery.

In the current study, a network meta-analysis was performed to comprehensively estimate the effects of different anesthesia manners, such as general anesthesia on the outcomes of orthopedic surgery. According to this analysis, we hope to provide some new insights for improving the outcomes of orthopedic surgery.

\section{Methods}

\section{Data sourcing}

According to the searching strategy, studies focused on the associations between anesthesia methods and adverse effects after orthopedic surgery published in English were downloaded from the Pubmed (http:// www.ncbi.nlm.nih.gov/pubmed), Embase (http:// www.embase.com), and the Cochrane Library (http:// www.cochranelibrary.com) databases. The searching date was ranged from its recording to Mar. 10th, 2018. The searching strategy was designed as follows: "general anesthesia" (OR "general anaesthesia" OR "local anesthesia" OR "topical anesthesia" OR "local anaesthesia" OR "toponarcosis" OR "medullary anesthesia" OR "rachianalgesia" OR "rachianesthesia" OR "medullary narcosis" OR "spinal anesthesia" OR "rhachiaesthesia" OR "rhachianalgesia" OR "lumbar anesthesia" OR "epidural anesthesia" OR "epidural block" OR "epidural anaesthesia" OR "caudal anaesthesia" OR "caudal anesthesia" OR "caudalanaesthesia" OR "infiltration analgesia" OR "intrathecal analgesia") AND "orthopedics" (OR "orthopedic" OR "osteology") AND "Rando".

\section{Inclusive and exclusive criteria}

In the present study, studies were included if they met the following terms: (1) published in English; (2) reported on the influences of different anesthesia methods on the effective of patients (P) undergoing orthopedic surgery; (3) patients in different groups receiving different anesthesia methods (Intervention, I; and Control, C); (4) study outcome variables including PONV, urine retention, back pain, sore throat, and headache, and so on (Outcomes, O); and (5) randomized controlled trial (RCT; S). Studies were excluded if they were met the following criteria: (1) incomplete data which could not be used for statistical analysis; (2) reviews, letters and comments; (3) for duplicate publication or data used for several studies, only the study with complete data was included, and others were excluded.

\section{Data extraction and quality assessment}

Data was independently extracted from the included studies by two censors in this study, respectively. The extracted information included the first author, published year, study year, study area, anesthesia method, sample size in different groups, length of operation, and the demographic characteristics of included patients, including age, gender, height, weight and so on. Quality of the enrolled studies were assessed using the Cochrane Collaboration recommendations recommended by the Cochrane system [11]. During the data extraction and quality assessment, divergences were solved by discussing with the third censors.

\section{Statistical analyses}

ADDIS is a non-programming software based on Bayesian framework, and can be used for data evaluation using the Markov chain Monte Carlo theory [12, 13]. All data in the current study was analyzed using the ADDIS software (version 1.16.5), and presented with odd ratio (OR) and 95\% confidence interval (CI). For $P<0.05$ in node-splitting analysis, the random effects model was used to calculate pooled effect size; otherwise, the consistency model were used to calculate the pooled effect size. Convergence degree of model was estimated using Brooks-Gelman-Rubin method, and presented with the potential scale reduction factor (PSRF). The more PSRF approximate to 1 , the better convergence was obtained [14].

\section{Results}

\section{Characteristics of enrolled studies}

According to the searching strategy, a total of 3196 studies were recruited in this study. After removing the repetitions, 1945 studies were obtained. Following this, 1779 papers among 1945 were rejected after scanning title and abstract. Subsequently, 143 studies among remains were removed after reviewing the full text. Finally, 23 studies were obtained [15-37] and the process of study enrollment was presented in Fig. 1A.

Characteristics of the enrolled studies were summarized in Table 1. For these enrolled studies, the published years of them were ranged from 1978 to 2017, and the research areas were concentrated on Germany, Japan, America, China, French, and Turkey. A total of 2393 patients were enrolled in this study, including 753 in general anesthesia (GA) group, 215 in epidural anesthesia (EA) group, 473 in local infiltration analgesia (LIA) group, 238 in nerve block analgesia (NBA) group, 


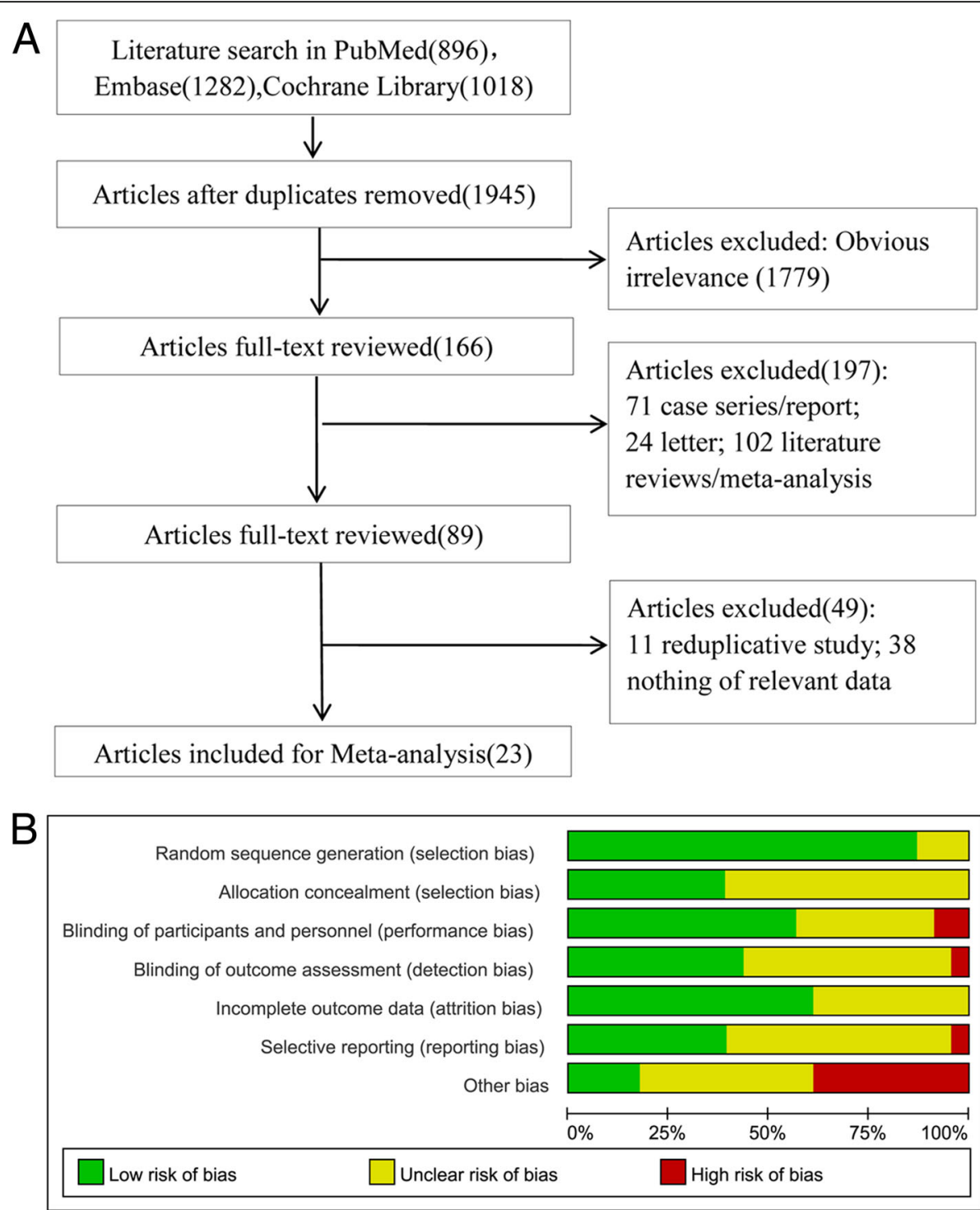

Fig. 1 Study enrollment and quality assessment. a, Flow chart of study enrollment; $\mathbf{b}$, Quality assessment of enrolled studies

630 in spinal anesthesia (SA) group, 65 in interscalene block (ISB) group, and 81 in GA + ISB group. Moreover, the number of male patients was slightly higher than female patients, but there was no significantly difference for gender ratio in each study. In addition, there were no significantly difference identified in the age, height, weight, and length of operation among groups. Quality assessment indicated that the enrolled studies presented an ordinary quality (Fig. 1B). Despite the random sequence generation (selection bias), most of studies didn't report more information on other quality assessment terms.

\section{Network meta-analyses for adverse effects after orthopedic surgery}

According to the extracted data, parameters of ADDIS were set as follows: Number of chains: 4, Tuning iterations: 20000, Simulation iterations: 50000, Thinning interval: 10, Inference samples: 10000, Variance scaling factor: 2.5, and the network meta-analyses for PONV, urine retention, sore throat, back pain and headache were analyzed.

\section{Network analysis for PONV}

For PONV, the PSRF value was ranged from 1.00 to 1.01, indicating model had a good convergence. The node-splitting analysis presented that $P$ values of all comparisons were more than 0.05 (Table 2A), and the consistency model was used to calculate the pooled effect sizes. The result presented that NBA had lowest influence on PONV after orthopedic surgery, and GA presented the worst effect on PONV after orthopedic surgery (Fig. 2A). Compared with NBA group, SA (OR 
Table 1 Characteristics of enrolled studies

\begin{tabular}{|c|c|c|c|c|c|c|c|c|c|c|}
\hline Author & $\begin{array}{l}\text { Public } \\
\text { Year }\end{array}$ & Location & Study Yeat & Group & N & $\begin{array}{l}\text { Age } \\
\text { (years)* }\end{array}$ & $\begin{array}{l}\text { Male/ } \\
\text { Female }\end{array}$ & Weight $(\mathrm{kg})^{*}$ & Height $(\mathrm{cm})^{*}$ & $\begin{array}{l}\text { Length of operation } \\
(\mathrm{min})^{*}\end{array}$ \\
\hline \multirow[t]{2}{*}{ Arcioni R } & \multirow[t]{2}{*}{2007} & \multirow[t]{2}{*}{ Italy } & \multirow{2}{*}{$\begin{array}{l}2004.9- \\
2004.12\end{array}$} & EA & 25 & $59.1 \pm 19.4$ & $12 / 13$ & $68.0 \pm 8.7$ & $166.2 \pm 8.4$ & $\mathrm{NA}$ \\
\hline & & & & SA & 23 & $60.2 \pm 21.2$ & $9 / 14$ & $68.3 \pm 10.4$ & $164.7 \pm 7.9$ & NA \\
\hline \multirow[t]{2}{*}{ Kuchalik J } & \multirow[t]{2}{*}{2013} & \multirow[t]{2}{*}{ Sweden } & \multirow[t]{2}{*}{ NA } & SA & 39 & $66(51-84)$ & $23 / 16$ & $84 \pm 23$ & $170 \pm 10$ & $106 \pm 17$ \\
\hline & & & & LIA & 39 & $67(50-85)$ & $21 / 18$ & $86 \pm 20$ & $173 \pm 8$ & $112 \pm 28$ \\
\hline \multirow[t]{2}{*}{ Dadure C } & \multirow[t]{2}{*}{2006} & \multirow[t]{2}{*}{ France } & \multirow{2}{*}{$\begin{array}{l}2001.7- \\
2002.12\end{array}$} & EA & 27 & $1-12$ & NA & $7-56$ & $72-160$ & 65-190 \\
\hline & & & & NBA & 25 & $1-11$ & NA & $10-52$ & $80-151$ & $45-180$ \\
\hline \multirow[t]{2}{*}{ Dunn WR } & \multirow[t]{2}{*}{2006} & \multirow[t]{2}{*}{ USA } & \multirow[t]{2}{*}{ NA } & LIA & 18 & Mean:51 & $11 / 7$ & Mean:75.0 & Mean:170.3 & Mean:27.4 \\
\hline & & & & SA & 14 & Mean:55 & $5 / 9$ & Mean:74.2 & Mean:169.6 & Mean:30.9 \\
\hline \multirow[t]{2}{*}{ Hadzic A } & \multirow[t]{2}{*}{2005} & \multirow[t]{2}{*}{ USA } & \multirow{2}{*}{$\begin{array}{l}2000.4- \\
2002.3\end{array}$} & ISB & 25 & $49 \pm 13$ & $17 / 8$ & $85 \pm 20$ & $173 \pm 10$ & $127 \pm 35$ \\
\hline & & & & GA & 25 & $49 \pm 12$ & $13 / 12$ & $86 \pm 21$ & $172 \pm 10$ & $147 \pm 49$ \\
\hline \multirow[t]{2}{*}{ Hadzic A } & \multirow[t]{2}{*}{2004} & \multirow[t]{2}{*}{ USA } & NA & NBA & 25 & $45 \pm 15$ & $12 / 13$ & $81 \pm 18$ & $173 \pm 10$ & $72(50-165)$ \\
\hline & & & & GA & 25 & $40 \pm 16$ & $11 / 14$ & $77 \pm 15$ & $170 \pm 10$ & $70(30-330)$ \\
\hline Janssen $\mathrm{H}$ & 2014 & Germany & NA & GA & 42 & $51 \pm 10$ & $19 / 23$ & $80 \pm 14$ & $170 \pm 7$ & $56.0 \pm 12.4$ \\
\hline & & & & $\begin{array}{l}\mathrm{GA}+ \\
\mathrm{ISB}\end{array}$ & 41 & $53 \pm 9$ & $18 / 23$ & $81 \pm 16$ & $170 \pm 8$ & $46.0 \pm 15.3$ \\
\hline Karaarslan S & 2015 & Turkey & NA & SA & 30 & $43 \pm 13$ & $13 / 17$ & $75 \pm 13$ & $170 \pm 7$ & $79 \pm 22$ \\
\hline & & & & NBA & 30 & $43 \pm 10$ & 19/11 & $77 \pm 16$ & $169 \pm 9$ & $85 \pm 23$ \\
\hline Krobbuaban & 2005 & Thailand & NA & SA & 86 & $41 \pm 20$ & $45 / 41$ & $58 \pm 13$ & $161 \pm 8$ & $86 \pm 52$ \\
\hline & & & & GA & 85 & $38 \pm 17$ & $47 / 38$ & $56 \pm 8$ & $168 \pm 7$ & $71 \pm 15$ \\
\hline Lehmann $\sqcup$ & 2014 & Germany & 2011.7- & GA & 40 & $54.1 \pm 11.7$ & $22 / 18$ & $83.3 \pm 16.6$ & $172.6 \pm 10.7$ & NA \\
\hline & & & & ISB & 40 & $49.3 \pm 13.6$ & $27 / 13$ & $88.2 \pm 19.2$ & $172.2 \pm 9.9$ & NA \\
\hline & & & & $\begin{array}{l}\mathrm{GA}+ \\
\mathrm{ISB}\end{array}$ & 40 & $53.8 \pm 15.2$ & $18 / 22$ & $81.5 \pm 16.3$ & $169 \pm 9.8$ & NA \\
\hline Nagafuchi M & 2015 & Japan & 2012.10- & NBA & 17 & $72 \pm 10$ & $2 / 15$ & $55 \pm 8.2$ & NA & $71 \pm 15$ \\
\hline & & & & LIA & 16 & $73 \pm 5.9$ & $3 / 13$ & $62 \pm 12.5$ & NA & $81 \pm 20$ \\
\hline Seeberger & 1994 & Switzerland & NA & SA & 96 & $33.7 \pm 12.3$ & $73 / 23$ & $73.7 \pm 12.6$ & $174.2 \pm 5.3$ & $62 \pm 35$ \\
\hline & & & & EA & 96 & $32.0 \pm 9.0$ & $67 / 29$ & $72.7 \pm 11.0$ & $174.7 \pm 5.4$ & $68 \pm 46$ \\
\hline Spangehl MJ & 2015 & NA & NA & NBA & 79 & $67.8 \pm 7.9$ & $41 / 37$ & NA & NA & NA \\
\hline & & & & LIA & 81 & $67.7 \pm 7.2$ & $48 / 43$ & NA & NA & NA \\
\hline Standl T & 1996 & Germany & NA & SA & 221 & $41.3 \pm 17.8$ & $112 / 109$ & $70.4 \pm 11$ & $170.5 \pm 8$ & $120 \pm 19$ \\
\hline & & & & GA & 212 & $43.2 \pm 17.3$ & 106/106 & $70.9 \pm 9$ & $172.1 \pm 6$ & $116 \pm 5$ \\
\hline Gi E & 2014 & Japan & NA & LIA & 25 & $77 \pm 7$ & $24 / 1$ & $61 \pm 13$ & $149 \pm 7$ & $174 \pm 23$ \\
\hline & & & & NBA & 24 & $78 \pm 5$ & $21 / 3$ & $64 \pm 13$ & $151 \pm 7$ & $173 \pm 27$ \\
\hline Bigler D & 1985 & NA & NA & GA & 20 & $77.6 \pm 2.3$ & $5 / 15$ & NA & NA & $59 \pm 10$ \\
\hline & & & & SA & 20 & $80.1 \pm 1.6$ & $2 / 18$ & NA & NA & $67 \pm 8$ \\
\hline Hole A & 1980 & Norway & NA & GA & 31 & $71.7(61-82)$ & $11 / 20$ & NA & NA & $207 \pm 6$ \\
\hline & & & & EA & 29 & $69.9(56-84)$ & $10 / 19$ & NA & NA & $190 \pm 6$ \\
\hline Kudoh A & 2004 & Japan & NA & SA & 75 & $75.9 \pm 4.0$ & $69 / 6$ & $60.4 \pm 8.7$ & $151.3 \pm 7.3$ & $106.7 \pm 31.5$ \\
\hline & & & & GA & 75 & $75.1 \pm 4.2$ & $66 / 9$ & $59.2 \pm 5.9$ & $149.3 \pm 5.4$ & $104.2 \pm 11.8$ \\
\hline McLaren AD & 1978 & UK & NA & GA & 29 & $76 \pm 9.7$ & NA & NA & NA & NA \\
\hline & & & & SA & 26 & $75.6 \pm 10.3$ & NA & NA & NA & NA \\
\hline Tanikawa H & 2014 & Japan & NA & LIA & 23 & $71(69-76)$ & $19 / 4$ & $\begin{array}{l}55.0(53.5- \\
66.0)\end{array}$ & 151(148-152) & $82.4 \pm 26.0$ \\
\hline & & & & NBA & 23 & $\begin{array}{l}72(67.5- \\
76.5)\end{array}$ & $20 / 3$ & $\begin{array}{l}54.5(48.0- \\
66.5)\end{array}$ & $\begin{array}{l}150(143.5- \\
155.5)\end{array}$ & $75.0 \pm 27.3$ \\
\hline
\end{tabular}


Table 1 Characteristics of enrolled studies (Continued)

\begin{tabular}{|c|c|c|c|c|c|c|c|c|c|c|}
\hline Author & $\begin{array}{l}\text { Public } \\
\text { Year }\end{array}$ & Location & Study Yeat & Group & $\mathrm{N}$ & $\begin{array}{l}\text { Age } \\
\text { (years)* }^{*}\end{array}$ & $\begin{array}{l}\text { Male/ } \\
\text { Female }\end{array}$ & Weight $(\mathrm{kg})^{*}$ & Height $(\mathrm{cm})^{*}$ & $\begin{array}{l}\text { Length of operation } \\
(\mathrm{min})^{*}\end{array}$ \\
\hline \multirow[t]{2}{*}{ Trker G } & \multirow[t]{2}{*}{2003} & \multirow[t]{2}{*}{ Turkey } & \multirow[t]{2}{*}{ NA } & EA & 15 & $62.2 \pm 6.6$ & $9 / 6$ & $72.2 \pm 7.5$ & $166.6 \pm 3$ & $129.2 \pm 26.4$ \\
\hline & & & & NBA & 15 & $62.3 \pm 7.2$ & $8 / 7$ & $73.7 \pm 6.3$ & $167.4 \pm 4.4$ & $131.3 \pm 18.7$ \\
\hline \multirow[t]{2}{*}{ Wang $\mathrm{H}$} & \multirow[t]{2}{*}{2017} & \multirow[t]{2}{*}{ China } & \multirow{2}{*}{$\begin{array}{l}2008.1- \\
2015.12\end{array}$} & GA & 169 & $52.9 \pm 9.7$ & $89 / 80$ & NA & NA & $52.5 \pm 9.3$ \\
\hline & & & & LIA & 187 & $51.4 \pm 9.1$ & $93 / 94$ & NA & NA & $48.1 \pm 9.9$ \\
\hline \multirow[t]{2}{*}{ Yukawa Y } & \multirow[t]{2}{*}{2005} & \multirow[t]{2}{*}{ Japan } & \multirow[t]{2}{*}{ NA } & LIA & 22 & $58.9 \pm 14.5$ & $15 / 7$ & $60.3 \pm 9.5$ & $159.2 \pm 7.9$ & $160.7 \pm 27.0$ \\
\hline & & & & EA & 23 & $59.1 \pm 15.2$ & $10 / 13$ & $59.0 \pm 9.7$ & $160.1 \pm 8.7$ & $157.5 \pm 29.5$ \\
\hline
\end{tabular}

Abbreviations: PONV: post-operative nausea or vomiting; GA: general anesthesia; LIA: local infiltration analgesia; ISB: interscalene block; EA: epidural anesthesia; NBA: nerve block analgesia; SA: spinal anesthesia; min: minutes; ${ }^{*}$ : mean \pm standard deviation/median $(\min -\max )$

$=0.31,95 \% \mathrm{CI}: 0.10,0.86)$, EA $(\mathrm{OR}=0.17,95 \% \mathrm{CI}: 0.06-$ $0.39)$, GA (OR $=0.07,95 \% \mathrm{CI}: 0.02-0.18)$, and $\mathrm{GA}+\mathrm{IBS}$ $(\mathrm{OR}=0.19,95 \% \mathrm{CI}: 0.04-0.81)$ presented significantly worse effect on PONV after orthopedic surgery (Table 3A).

\section{Network analysis for urine retention}

The PSRF for urine retention was ranged from 1.00 to 1.02 indicating a good convergence for PSRF. Node-splitting analysis presented that $P>0.05$, thus, the consistency model was used to calculate the pooled effect size of urine retention (Table 2B). The network analysis presented that the NBA group presented the lowest incidence of urine retention, and its incidence was significantly lower than that in the EA group $(\mathrm{OR}=0.07$, 95\%CI: 0.01-0.37, Table 3B and Fig. 2B).

\section{Analysis for sore throat}

For sore throat, all PSRF values were 1.01, indicating a good convergence. Because no closed ring formed, consistency model was utilized to calculate the pooled side effect of sore throat. The analytical results presented that both the SA and NBA groups had lower incidences of sore throat, but no significant differences were identified compared with other groups (Fig. 3, Table 4).

\section{Analysis for back pain}

For back pain, all PSRF values were 1.01, indicating a good convergence. Because no closed ring formed, consistency model was utilized to calculate the pooled side effect of back pain. Compared with the EA group, both ISB (OR $=0.00,95 \% \mathrm{CI}: 0.00-0.30)$ and LIA (OR = 0.00 , 95\%CI: $0.00-0.25)$ groups presented lower

Table 2 Node-splitting analysis for PONV and urine retention

\begin{tabular}{|c|c|c|c|c|}
\hline Name & Direct Effect & Indirect Effect & Overall & $P$-Value \\
\hline \multicolumn{5}{|l|}{ A: PONV } \\
\hline$E A, G A$ & $1.02(-0.47,2.42)$ & $0.88(-0.31,2.09)$ & $0.91(0.02,1.88)$ & 0.89 \\
\hline$E A, S A$ & $0.25(-1.15,1.85)$ & $-1.24(-2.46,-0.20)$ & $-0.68(-1.58,0.25)$ & 0.10 \\
\hline$E A, L I A$ & $-2.46(-4.33,-0.74)$ & $-1.38(-2.44,-0.38)$ & $-1.74(-2.67,-0.89)$ & 0.29 \\
\hline EA, NBA & $-2.03(-4.03,-0.76)$ & $-1.46(-2.79,-0.21)$ & $-1.80(-2.82,-0.93)$ & 0.47 \\
\hline GA, LIA & $-2.43(-4.62,-0.74)$ & $-2.77(-3.90,-1.76)$ & $-2.64(-3.70,-1.75)$ & 0.78 \\
\hline GA, NBA & $-1.80(-4.09,-0.11)$ & $-2.95(-4.22,-1.99)$ & $-2.71(-3.88,-1.74)$ & 0.41 \\
\hline $\mathrm{GA}, \mathrm{SA}$ & $-1.78(-2.62,-0.96)$ & $-0.91(-2.41,0.54)$ & $-1.57(-2.27,-0.88)$ & 0.30 \\
\hline$G A+I S B, I S B$ & $-1.03(-3.23,0.72)$ & $-1.62(-3.68,0.32)$ & $-1.23(-2.76,0.23)$ & 0.65 \\
\hline LIA, SA & $0.83(-0.50,2.13)$ & $1.29(0.13,2.66)$ & $1.08(0.20,2.04)$ & 0.62 \\
\hline LIA, NBA & $-0.08(-0.90,0.65)$ & $0.04(-1.68,1.49)$ & $-0.07(-0.81,0.63)$ & 0.89 \\
\hline \multicolumn{5}{|l|}{ B: Urine retention } \\
\hline$E A, G A$ & $-0.47(-2.91,1.86)$ & $-1.37(-4.68,1.53)$ & $-0.68(-2.52,0.87)$ & 0.60 \\
\hline$E A, N B A$ & $-2.93(-5.49,-0.99)$ & $-1.35(-5.92,2.38)$ & $-2.59(-4.56,-1.00)$ & 0.45 \\
\hline$E A, S A$ & $-0.66(-4.75,2.23)$ & $-0.67(-3.34,1.44)$ & $-0.76(-2.71,0.86)$ & 0.96 \\
\hline GA, SA & $0.20(-1.68,2.06)$ & $-0.94(-4.47,2.55)$ & $-0.08(-1.60,1.43)$ & 0.55 \\
\hline NBA, SA & $0.81(-2.34,4.35)$ & $2.50(-0.56,5.47)$ & $1.84(-0.26,3.93)$ & 0.48 \\
\hline
\end{tabular}

Abbreviations: PONV: post-operative nausea or vomiting; GA: general anesthesia; LIA: local infiltration analgesia; ISB: interscalene block; EA: epidural anesthesia; NBA: nerve block analgesia; SA: spinal anesthesia. Data was presented with odds ratio and $95 \%$ confidence interval 


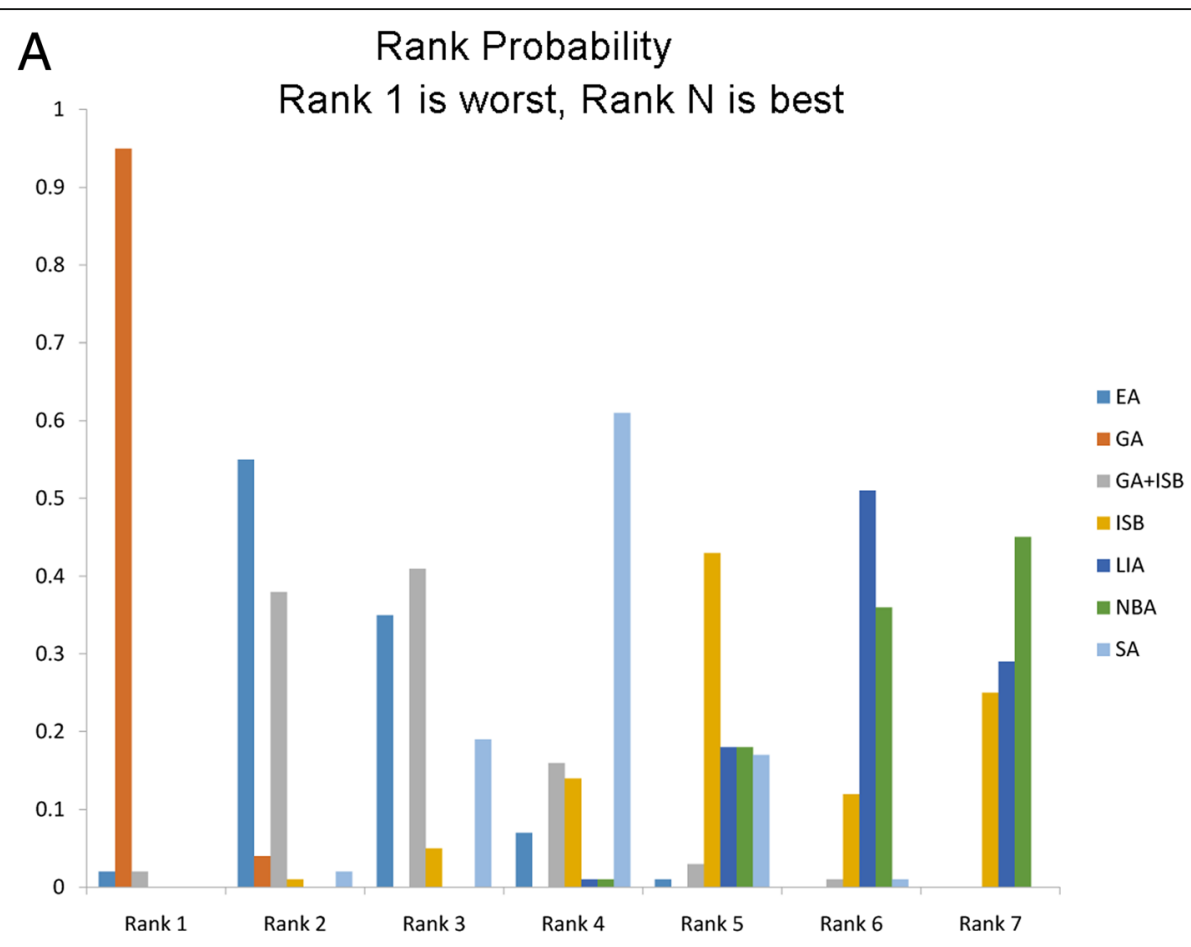

B

Rank Probability

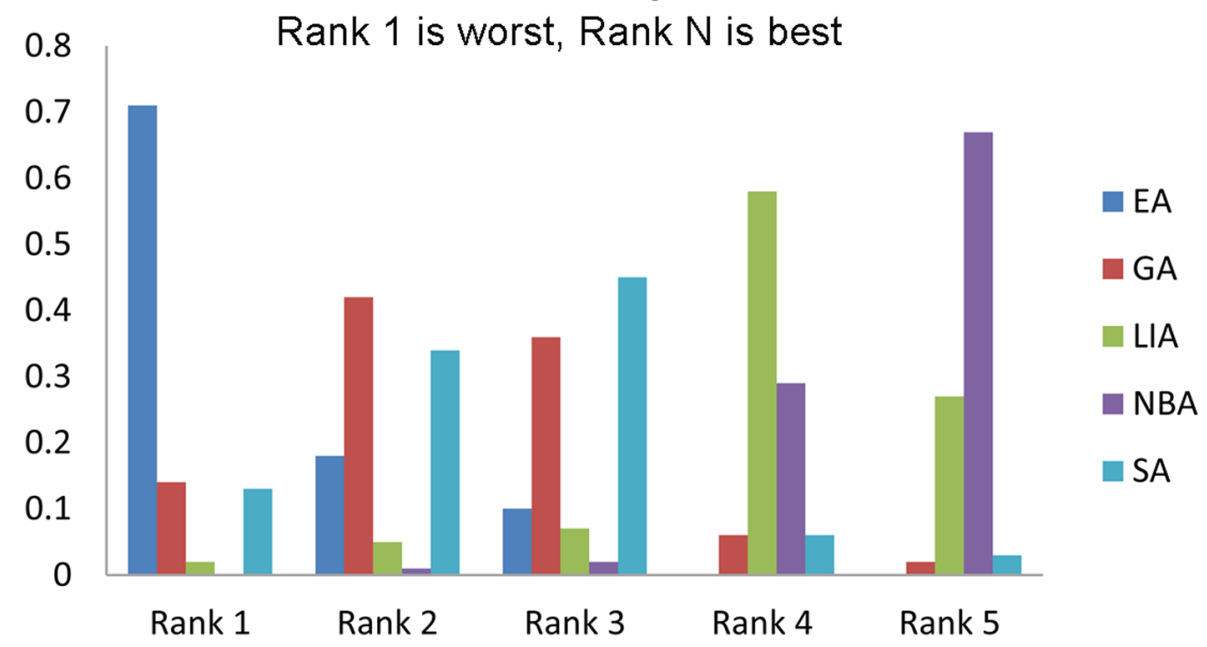

Fig. 2 Network meta-analyses for PONV and urine retention. a, Network meta-analyses for PONV; $\mathbf{b}$, Network meta-analyses for urine retention. PONV: post-operative nausea or vomiting; GA: general anesthesia; LIA: local infiltration analgesia; ISB: interscalene block; EA: epidural anesthesia; NBA: nerve block analgesia; SA: spinal anesthesia

incidences of back pain; however, no other significant difference was identified in comparison between other groups (Fig. 4A, Table 5A).

\section{Analysis for headache}

For back pain, all PSRF values were 1.01, indicating a good convergence. Because no closed ring formed, consistency model was utilized to calculate the pooled side effect of back pain. The network analysis presented that LIA group had the lowest incidence of headache, but no significant difference was revealed compared with other groups (Fig. 4B, Table 5B).

\section{Discussion}

According to the selective criteria, a total of 23 studies with 2393 patients were enrolled in this study. With the network meta-analysis, patients undergoing NBA presented lower occurrence rates of PONV and urine 
Table 3 Network meta-analyses for PONV and urine retention

\begin{tabular}{|c|c|c|c|c|c|c|}
\hline \multicolumn{7}{|l|}{ A: PONV } \\
\hline EA & $2.48(1.02,6.55)$ & $0.85(0.23,3.54)$ & $0.26(0.05,1.17)$ & $0.18(0.07,0.41)$ & $0.17(0.06,0.39)$ & $0.51(0.21,1.29)$ \\
\hline $0.40(0.15,0.98)$ & GA & $0.34(0.13,0.97)$ & $0.10(0.03,0.36)$ & $0.07(0.02,0.17)$ & $0.07(0.02,0.18)$ & $0.21(0.10,0.42)$ \\
\hline $1.17(0.28,4.42)$ & $2.91(1.03,7.79)$ & $G A+I S B$ & $0.29(0.06,1.25)$ & $0.20(0.05,0.76)$ & $0.19(0.04,0.81)$ & $0.59(0.17,1.94)$ \\
\hline $3.81(0.86,18.19)$ & $9.67(2.78,36.55)$ & $3.42(0.80,15.77)$ & ISB & $0.68(0.14,3.40)$ & $0.63(0.11,3.34)$ & $2.02(0.48,8.87)$ \\
\hline $5.71(2.44,14.43)$ & $13.98(5.74,40.29)$ & $4.91(1.32,21.15)$ & $1.48(0.29,7.31)$ & LIA & $0.93(0.44,1.87)$ & $2.95(1.22,7.69)$ \\
\hline $6.02(2.53,16.79)$ & $15.08(5.69,48.64)$ & $5.24(1.24,25.69)$ & $1.58(0.30,8.71)$ & $1.07(0.53,2.26)$ & NBA & $3.22(1.16,9.67)$ \\
\hline $1.97(0.78,4.85)$ & $4.82(2.40,9.66)$ & $1.69(0.52,5.87)$ & $0.49(0.11,2.08)$ & $0.34(0.13,0.82)$ & $0.31(0.10,0.86)$ & SA \\
\hline \multicolumn{7}{|l|}{ B: Urine retention } \\
\hline EA & $0.51(0.08,2.38)$ & $0.10(0.01,1.18)$ & & $0.07(0.01,0.37)$ & $0.47(0.07,2.37)$ & \\
\hline $1.98(0.42,12.43)$ & GA & $0.21(0.01,3.65)$ & & $0.15(0.02,1.36)$ & $0.92(0.20,4.18)$ & \\
\hline $9.84(0.84,151.97)$ & $4.87(0.27,82.03)$ & LIA & & $0.71(0.11,5.12)$ & $4.34(0.25,77.47)$ & \\
\hline $13.36(2.73,95.12)$ & $6.83(0.74,57.95)$ & $1.41(0.20,9.26)$ & & NBA & $6.27(0.77,51.01)$ & \\
\hline $2.14(0.42,15.04)$ & $1.09(0.24,4.94)$ & $0.23(0.01,3.95)$ & & $0.16(0.02,1.29)$ & SA & \\
\hline
\end{tabular}

PONV: post-operative nausea or vomiting; GA: general anesthesia; LIA: local infiltration analgesia; ISB: interscalene block; EA: epidural anesthesia; NBA: nerve block analgesia; SA: spinal anesthesia. Data was presented with odds ratio and $95 \%$ confidence interval

retention compared with patients managed with SA, EA, GA, and GA + ISB during the perioperative period of orthopedic surgery. Meanwhile, patients managed with ISB and LIA were presented a significant lower occurrence rate of back pain compared with patients undergoing EA. However, there was no significant difference identified in the occurrence of headache among these groups.

NBA is a common anesthesia method utilized in orthopedic surgery, such as total knee arthroplasty [38], shoulder arthroscopy [39], and hip fracture [40]. It has been revealed that nerve blocks may present some benefits in lower risk of PONV, enhanced pain relief and earlier discharge [41, 42]. Park et al. have demonstrated that interscalene brachial plexus block could significantly reduce the nausea and vomiting, while suprascapular nerve anesthesia and intra-articular local anesthesia can't reduce the nausea and vomiting compared with the non-pain controlled group [43]. Hadzic et al. have identified that NBA can reduce the PONV compared with the general anesthesia for patients undergoing outpatients rotator cuff surgery [44]. During podiatric surgery in children, patients managed with EA present a higher risks for adverse events, including PONV and urine retention [18]. However, a previous meta-analysis has summarized that patients managed with NBA present a

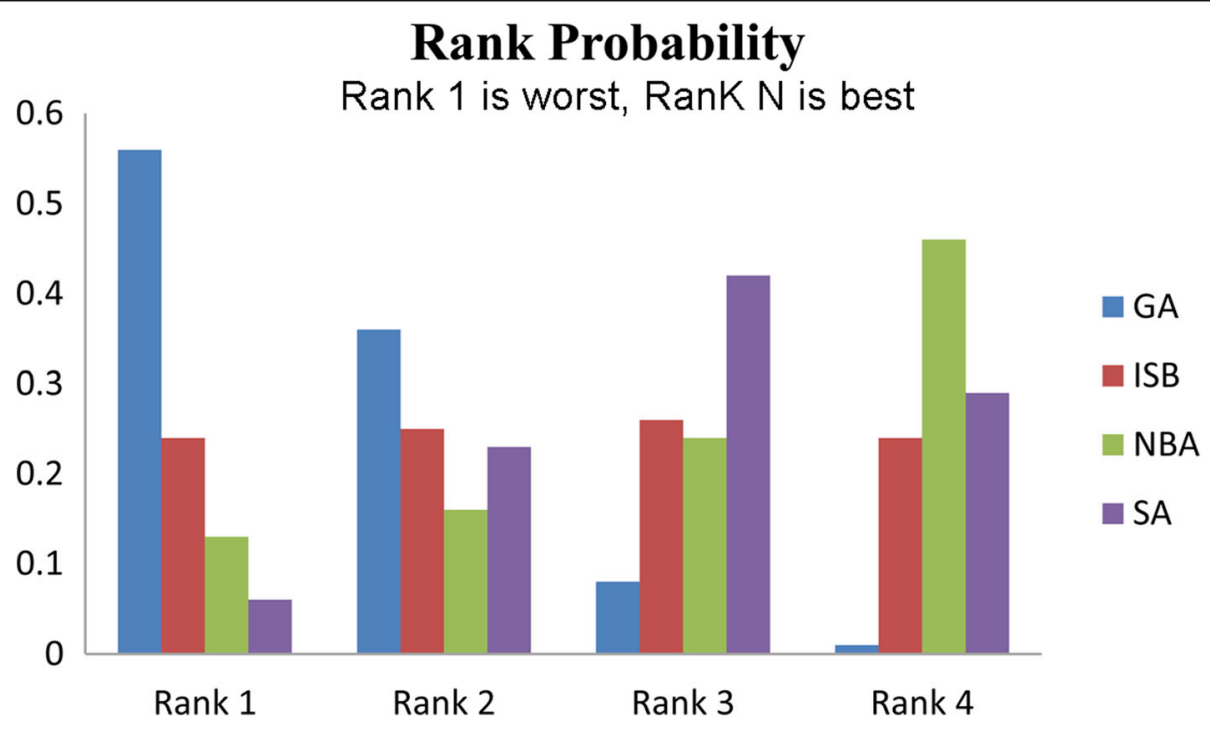

Fig. 3 Network meta-analyses for sore throat. GA: general anesthesia; LIA: local infiltration analgesia; ISB: interscalene block; NBA: nerve block analgesia; SA: spinal anesthesia 
Table 4 Network meta-analysis for sore throat

\begin{tabular}{llll}
\hline GA & $0.18(0.00,107.05)$ & $0.05(0.00,36.33)$ & $0.09(0.00,4.78)$ \\
\hline $5.43(0.01,3647.30)$ & ISB & $0.25(0.00,2894.67)$ & $0.48(0.00,868.90)$ \\
$20.89(0.03,21,288.40)$ & $3.93(0.00,56,736.22)$ & NBA & $1.74(0.00,5921.00)$ \\
$11.51(0.21,588.42)$ & $2.09(0.00,4009.84)$ & $0.57(0.00,1205.54)$ & SA \\
\hline
\end{tabular}

GA: general anesthesia; ISB: interscalene block; NBA: nerve block analgesia; SA: spinal anesthesia. Data was presented with odds ratio and 95\% confidence interval

lower incidence of urine retention than patients undergoing EA, but there is no difference in the incidence of PONV [45]. With an updated meta-analysis, NBA was identified to put a significant lower effects on the occurrence rates of PONV, urine retention, and sore throat compared with patients managed with SA, EA, GA, and GA + ISB during the perioperative period of orthopedic surgery in the current study. All of these findings indicated that NBA might perform a better outcome on the prognosis of patients undergoing orthopedic surgery.
ISB is one of the most reliable and commonly anesthetic method applied for the upper extremity with less opioid consumption and opioid-associated adverse effect [46]. Meanwhile, LIA is a safety and effective method for pain control during the perioperative periods of knee and hip surgery [47]. In this study, patients managed with ISB and LIA presented a significant lower occurrence rate of back pain compared with patients undergoing EA, indicating that ISB and LIA might play a better outcome for relieving back pain during orthopedic surgery. Adersen et al. have revealed that LIA

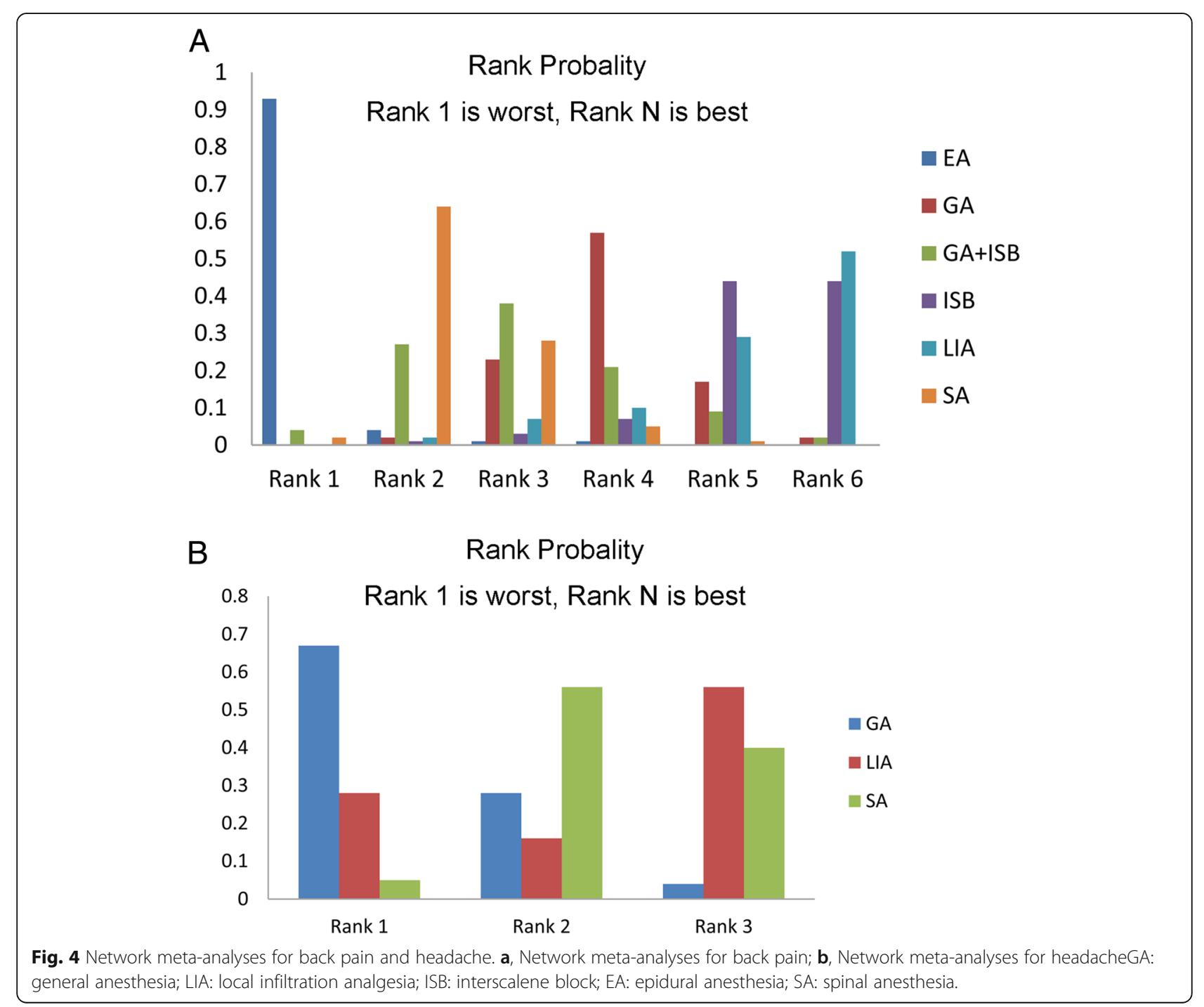


Table 5 Network meta-analyses for back pain and headache

\begin{tabular}{|c|c|c|c|c|c|}
\hline \multicolumn{6}{|l|}{ A: Back pain } \\
\hline EA & $0.02(0.00,0.57)$ & $0.04(0.00,2.38)$ & $0.00(0.00,0.30)$ & $0.00(0.00,0.25)$ & $0.06(0.00,1.38)$ \\
\hline $51.32(1.75,3339.72)$ & GA & $1.79(0.14,23.31)$ & $0.20(0.01,3.35)$ & $0.18(0.00,5.98)$ & $2.98(0.75,20.40)$ \\
\hline $27.51(0.42,4086.82)$ & $0.56(0.04,7.20)$ & $G A+I S B$ & $0.12(0.01,1.81)$ & $0.10(0.00,8.09)$ & $1.69(0.10,43.87)$ \\
\hline $267.22(3.36,44,447.31)$ & $4.89(0.30,104.69)$ & $8.53(0.55,176.80)$ & ISB & $0.82(0.01,96.19)$ & $15.26(0.73,591.84)$ \\
\hline $306.91(3.94,62,665.12)$ & $5.69(0.17,374.21)$ & 10.33(0.12,1263.02) & $1.22(0.01,173.76)$ & LIA & $17.27(0.82,906.94)$ \\
\hline $15.74(0.72,650.35)$ & $0.34(0.05,1.33)$ & $0.59(0.02,9.74)$ & $0.07(0.00,1.37)$ & $0.06(0.00,1.22)$ & SA \\
\hline \multicolumn{6}{|l|}{ B: Headache } \\
\hline GA & $0.32(0.00,25.48)$ & & $0.42(0.10,1.60)$ & & \\
\hline $3.17(0.04,217.06)$ & LIA & & $1.39(0.02,63.62)$ & & \\
\hline $2.40(0.63,10.17)$ & $0.72(0.02,55.14)$ & & SA & & \\
\hline
\end{tabular}

GA: general anesthesia; LIA: local infiltration analgesia; ISB: interscalene block; EA: epidural anesthesia; SA: spinal anesthesia. Data was presented with odds ratio and $95 \%$ confidence interval

presents a superior outcome with less adverse effect, including pain control, than EA during total knee arthroplasty [48]. Another study has also demonstrated that LIA performs a better outcome in pain controlling during total knee arthroplasty [49]. These findings demonstrated LIA and ISB might perform effective roles in relieving pains, such as back pain, during the perioperative period of orthopedic surgery. Despite of these, LIA was also identified to play critical role in relieving headache during the perioperative period of orthopedic surgery, but no statistically difference was identified compared with other group. Therefore, further investigation with large sample size might be required.

Although this study was the first to compare the effects of different anesthesia methods on the complications of orthopedic surgery, but there were still some limitations in this study. First, due to the incomplete data in studies, correction of concomitant variables was not performed, which might affect the results identified in this study. Meanwhile, the subgroup analysis was also not conducted. Second, limited by the property of ADDIS, the calculation of pooled effect size might be influenced. Third, some complications, such as headache and back pain, were not reported in several anesthesia methods; thus, there might be some bias contained in this study.

\section{Conclusions}

In conclusion, according to the network analysis, NBA was a superior anesthesia method in reliving the occurrence of PONV, urine retention, and sore throat compared with patients managed with SA, EA, GA, and GA + ISB during the perioperative period of orthopedic surgery. ISB and LIA were two effective anesthesia methods in lowering the occurrence rate of back pain during the perioperative period of orthopedic surgery. Therefore, it is important to surgeons to select appropriate anesthesia methods during the perioperative period of orthopedic surgery according to the physical fitness of patients and the effects of anesthesia methods on the occurrence of complications.

\section{Abbreviations}

Cl: confidence interval; EA: epidural anesthesia; ISB: interscalene block; LIA: local infiltration analgesia; NBA: nerve block analgesia; OR: odd ratio; PSRF: potential scale reduction factor; SA: spinal anesthesia

\section{Acknowledgements}

Not applicable.

Funding

No funding was obtained for this study.

Availability of data and materials

The datasets used and/or analysed during the current study are available from the corresponding author on reasonable request.

\section{Authors' contributions}

$Y Z$, JW and SD conceived the research. HR and JL acquired the data. $Y Z$ and FZ performed the statistics analysis. YZ and JW drafted the manuscript. SD revised the manuscript. All authors have read and approved the manuscript.

Ethics approval and consent to participate

Not applicable.

Consent for publication

Not applicable.

Competing interests

The authors declare that they have no competing interests.

\section{Publisher's Note}

Springer Nature remains neutral with regard to jurisdictional claims in published maps and institutional affiliations.

\section{Author details}

${ }^{1}$ The First Clinical Medical College, Guangzhou University of Chinese Medicine, 16 Jichang Road, Baiyun District, Guangzhou 510405, Guangdong Province, People's Republic of China. ${ }^{2}$ Department of Orthopaedics, Tongde Hospital of Zhejiang Province, Hangzhou, Zhejiang Province, People's Republic of China. ${ }^{3}$ Department of Hematology, The First Affliated Hospital of Zhejiang Chinese Medical University, 54 Youdian Road, Hangzhou, Zhejiang Province, People's Republic of China. 


\section{Received: 25 November 2018 Accepted: 21 February 2019} Published online: 09 April 2019

\section{References}

1. Waheeb A, Zywiel MG, Palaganas M, Venkataramanan V, Davis AM. The influence of patient factors on patient-reported outcomes of orthopedic surgery involving implantable devices: a systematic review. Seminars in Arthritis \& Rheumatism. 2015;44:461-71.

2. Huang F, Wu D, Ma G, Yin Z, Wang Q. The use of tranexamic acid to reduce blood loss and transfusion in major orthopedic surgery: a meta-analysis. Transfusion \& Apheresis Science. 2014;186:318-27.

3. Andrade N, Schmiedt CW, Cornell K, Radlinsky MG, Heidingsfelder L, Clarke K, et al. Survey of intraoperative bacterial contamination in dogs undergoing elective orthopedic surgery. Veterinary Surgery Vs. 2016;45:214-22.

4. Veljkovic A, Dwyer T, Lau JT, Abbas KZ, Salat P, Brull R. Neurological complications related to elective orthopedic surgery: part 3: common foot and ankle procedures. Regional Anesthesia \& Pain Medicine. 2015;40:455

5. Hu N, Guo D, Wang H, Xie K, Wang C, Li Y, et al. Involvement of the bloodbrain barrier opening in cognitive decline in aged rats following orthopedic surgery and high concentration of sevoflurane inhalation. Brain Res. 2014; 1551:13-24.

6. Safavi M, Honarmand A, Negahban M. Attari M. Prophylactic effects of intrathecal Meperidine and intravenous Ondansetron on shivering in patients undergoing lower extremity orthopedic surgery under spinal anesthesia. 2014;3:94-9.

7. Pugely AJ, Martin CT, Gao Y, Mendozalattes S, Callaghan JJ. Differences in short-term complications between spinal and general anesthesia for primary Total knee arthroplasty. Jbjs. 2013;95:193-9.

8. Helwani MA, Avidan MS, Abdallah AB, Kaiser DJ, Clohisy JC, Hall BL, et al. Effects of regional versus general anesthesia on outcomes after Total hip arthroplasty. J Bone Joint Surg (Am Vol). 2015;97:186-93.

9. Stundner $O$, Chiu YL, Sun X, Mazumdar M, Fleischut P, Poultsides $L$, et al. Comparative perioperative outcomes associated with neuraxial versus general anesthesia for simultaneous bilateral total knee arthroplasty. Regional Anesthesia \& Pain Medicine. 2012;37:638.

10. Mason SE, Noel-Storr A, Ritchie CW. The impact of general and regional anesthesia on the incidence of post-operative cognitive dysfunction and post-operative delirium: a systematic review with meta-analysis: Centre for Reviews and Dissemination (UK); 2010. 67-79 p.

11. Higgins JE. Cochrane Handbook for Systematic Reviews of Interventions. http://wwwcochrane-handbookorg. 2008; 5: S38.

12. Hillege $H$, Brock BD, Valkenhoef GV, Zhao J. ADDIS: an automated way to do network meta-analysis. Research Report. 2016.

13. Van Valkenhoef $G$, Tervonen T, Zwinkels T, De Brock B, Hillege H. ADDIS: a decision support system for evidence-based medicine. Decis Support Syst. 2013:55:459-75

14. Brooks SP, Gelman A. General methods for monitoring convergence of iterative simulations. Journal of Computational \& Graphical Statistics. 1998;7:434-55.

15. Hole A, Terjesen T, Breivik H. Epidural versus general anaesthesia for total hip arthroplasty in elderly patients. Acta Anaesthesiol Scand. 1980;24:279-87.

16. Arcioni R, Palmisani S, Tigano S, Santorsola C, Sauli V, Romanò S, et al. Combined intrathecal and epidural magnesium sulfate supplementation of spinal anesthesia to reduce post-operative analgesic requirements: a prospective, randomized, double-blind, controlled trial in patients undergoing major orthopedic surgery. Acta Anaesthesiol Scand. 2007;51: 482-9.

17. Bigler D, Adelhøj B, Petring OU, Pederson NO, Busch P, Kalhke P. Mental function and morbidity after acute hip surgery during spinal and general anaesthesia. Anaesthesia. 1985;40:672-6.

18. Dadure C, Bringuier S, Nicolas F, Bromilow L, Raux O, Rochette A, et al. Continuous epidural block versus continuous popliteal nerve block fo postoperative pain relief after major podiatric surgery in children: a prospective, comparative randomized study. Anesth Analg. 2006;102:744

19. Dunn WR, Cordasco FA, Flynn E, Jules K, Gordon M, Liguori G. A prospective randomized comparison of spinal versus local anesthesia with Propofol infusion for knee arthroscopy. Arthroscopy the Journal of Arthroscopic \& Related Surgery. 2006;22:479-83.

20. Gi E, Yamauchi M, Yamakage M, Kikuchi C, Shimizu H, Okada Y, et al. Effects of local infiltration analgesia for posterior knee pain after total knee arthroplasty: comparison with sciatic nerve block. J Anesth. 2014;28:696-701.
21. Hadzic A, Arliss J, Kerimoglu B, Karaca PE, Yufa M, Claudio RE, et al. A comparison of infraclavicular nerve block versus general anesthesia for hand and wrist day-case surgeries. Anesthesiology. 2004;101:127-32.

22. Hadzic A, Williams BA, Karaca PE, Hobeika P, Unis G, Dermksian J, et al. For outpatient rotator cuff surgery, nerve block anesthesia provides superior sameday recovery over general anesthesia. Anesthesiology. 2005;102:1001-7.

23. Janssen H, Stosch RV, Pöschl R, Büttner B, Bauer M, Hinz JM, et al. Blood pressure response to combined general anaesthesia/interscalene brachial plexus block for outpatient shoulder arthroscopy. BMC Anesthesiol. 2014;14:50.

24. Karaarslan S, Tekgül ZT. E Ş, Turan M, Karaman Y, Kaya A, et al. Comparison Between Ultrasonography-Guided Popliteal Sciatic Nerve Block and Spinal Anesthesia for Hallux Valgus Repair. 2015;37.

25. Krobbuaban B, Kumkeaw S, Pakdeesirivong N, Diregpoke S. Comparison of postanesthetic complaints after general and spinal anesthesia in patients undergoing lower limb surgery. Journal of the medical Association of Thailand $=$. Chotmaihet thangphaet. 2005;88:909.

26. Kuchálik J, Granath B, Ljunggren A, Magnuson A, Lundin A, Gupta A. Postoperative pain relief after total hip arthroplasty: a randomized, doubleblind comparison between intrathecal morphine and local infiltration analgesia. Br J Anaesth. 2013;111:793-9.

27. Kudoh A, Takase H, Takazawa T. A comparison of anesthetic quality in propofol-spinal anesthesia and propofol-fentanyl anesthesia for total knee arthroplasty in elderly patients. J Clin Anesth. 2004;16:405.

28. Lehmann L, Loosen G, Weiss C, Schmittner MD. Interscalene plexus block versus general anaesthesia for shoulder surgery: a randomized controlled study. European Journal of Orthopaedic Surgery \& Traumatology. 2015;25:255.

29. Mclaren AD, Stockwell MC, Reid VT. Anaesthetic techniques for surgical correction of fractured neck of femur. A comparative study of spinal and general anaesthesia in the elderly. Anaesthesia. 1978;33:10-4.

30. Nagafuchi M, Sato T, Sakuma T, Uematsu A, Hayashi H. Tanikawa H, et al. Femoral nerve block-sciatic nerve block vs femoral nerve block-local infiltration analgesia for total knee arthroplasty: a randomized controlled trial Bmc Anesthesiology. 2015;15:182.

31. Seeberger MD, Lang ML, Drewe J, Schneider M, Hauser E, Hruby J. Comparison of spinal and epidural anesthesia for patients younger than 50 years of age. Anesth Analg. 1994;78:667-73.

32. Standl T, Eckert S, Schulteam EJ. Postoperative complaints after spinal and thiopentone-isoflurane anaesthesia in patients undergoing orthopaedic surgery. Spinal versus general anaesthesia. Acta Anaesthesiol Scand. 1996;40:222-6.

33. Tanikawa H, Sato T, Nagafuchi M, Takeda K, Oshida J, Okuma K. Comparison of local infiltration of analgesia and sciatic nerve block in addition to femoral nerve block for total knee arthroplasty. J Arthroplasty. 2014;29:2462-7.

34. Türker G, Uçkunkaya N, Yavaşçaoğlu B, Yilmazlar A, Ozçelik S. Comparison of the catheter-technique psoas compartment block and the epidural block for analgesia in partial hip replacement surgery. Acta Anaesthesiol Scand. 2010;47:30-6.

35. Wang H, Ma L, Yang D, Wang T, Wang Q, Zhang L, et al. Cervical plexus anesthesia versus general anesthesia for anterior cervical discectomy and fusion surgery: a randomized clinical trial. Medicine. 2017;96:e6119.

36. Yukawa $Y$, Kato F, Ito K, Terashima T, Horie Y. A prospective randomized study of preemptive analgesia for postoperative pain in the patients undergoing posterior lumbar interbody fusion: continuous subcutaneous morphine, continuous epidural morphine, and diclofenac sodium. Spine. 2005;30:2357.

37. Spangehl MJ, Clarke HD, Hentz JG, Misra L, Blocher JL, Seamans DP. The Chitranjan Ranawat Award: Periarticular Injections and Femoral \& Sciatic Blocks Provide Similar Pain Relief After TKA: A Randomized Clinical Trial. Clinical Orthopaedics and Related Research ${ }^{\oplus}$. 2015; 473: 45-53.

38. Ng FY, Chiu KY, Yan CH, Ng KF. Continuous femoral nerve block versus patientcontrolled analgesia following total knee arthroplasty. J Orthop Surg. 2012;20:23.

39. Chang KV, Hung CY, Wu WT, Han DS, Yang RS, Lin CP. Comparison of the effectiveness of suprascapular nerve block with physical therapy, placebo, and intra-articular injection in Management of Chronic Shoulder Pain- a meta-analysis of randomized controlled trials. Archives of Physical Medicine \& Rehabilitation. 2015:97:1366-80.

40. Beaudoin FL, Haran JP, Liebmann O. A comparison of ultrasound-guided three-in-one femoral nerve block versus parenteral opioids alone for analgesia in emergency department patients with hip fractures: a randomized controlled trial. Acad Emerg Med. 2013;20:584-91.

41. Junger A, Klasen J, Benson M, Sciuk G, Hartmann B, Sticher J, et al. Factors determining length of stay of surgical day-case patients. Eur J Anaesthesiol. 2001;18:314-21. 
42. Chung F, Ritchie E, Su J. Postoperative pain in ambulatory surgery. Anesth Analg. 1997;85:808-16.

43. Park SK, Choi YS, Choi SW, Song SW. A comparison of three methods for postoperative pain control in patients undergoing arthroscopic shoulder surgery. Korean J Pain. 2015;28:45-51.

44. Hadzic MDPDA, Arliss MDJ, Kerimoglu MDB, Karaca MD Pelin E, Yufa MDM, Claudio BS,Richard E, et al. A comparison of Infraclavicular nerve block versus general anesthesia for hand and wrist day-case surgeries. Anesthesiology. 2004; 101: 127-132.

45. Fowler SJ, Symons J, Sabato S, Myles PS. Epidural analgesia compared with peripheral nerve blockade after major knee surgery: a systematic review and meta-analysis of randomized trials. Br J Anaesth. 2008;100:154-64.

46. McNaught A, Shastri U, Carmichael N, Awad IT, Columb M, Cheung J, et al. Ultrasound reduces the minimum effective local anaesthetic volume compared with peripheral nerve stimulation for interscalene block. British Journal of Anaesthesia. 106: 124-130.

47. Kerr DR, Kohan L. Local infiltration analgesia: a technique for the control of acute postoperative pain following knee and hip surgery: a case study of 325 patients. Acta Orthop. 2008;79:174-83.

48. Andersen KV, Bak M, Christensen BV, Harazuk J, Pedersen NA, Søballe K. A randomized, controlled trial comparing local infiltration analgesia with epidural infusion for total knee arthroplasty. Acta Orthop. 2010;81:606-10.

49. Spreng UJ, Dahl V, Hjall A, Fagerland MW, Ræder J. High-volume local infiltration analgesia combined with intravenous or local ketorolac +morphine compared with epidural analgesia after total knee arthroplasty. Br J Anaesth. 2010;105:675-82.

Ready to submit your research? Choose BMC and benefit from:

- fast, convenient online submission

- thorough peer review by experienced researchers in your field

- rapid publication on acceptance

- support for research data, including large and complex data types

- gold Open Access which fosters wider collaboration and increased citations

- maximum visibility for your research: over $100 \mathrm{M}$ website views per year

At $\mathrm{BMC}$, research is always in progress.

Learn more biomedcentral.com/submissions 